\title{
A Matching Degree Management Model of Human Body Shape and Fashion Design Based on Big Data Analysis
}

\author{
Yumei Cui, Xinqun Feng $\mathbb{D}^{\mathbb{D}}$, and Xinxin Yang \\ College of Fashion and Design, Donghua University, Shanghai 200051, China \\ Correspondence should be addressed to Xinxin Yang; 1169189@mail.dhu.edu.cn
}

Received 15 September 2021; Accepted 22 October 2021; Published 8 November 2021

Academic Editor: Tongguang Ni

Copyright (c) 2021 Yumei Cui et al. This is an open access article distributed under the Creative Commons Attribution License, which permits unrestricted use, distribution, and reproduction in any medium, provided the original work is properly cited.

The existing clothing design model lacks the screening link of the human body part index, and the output clothing data are affected by the high correlation coefficient, resulting in large matching errors. Therefore, based on the analysis of human body shape, a management model of matching degree of human body shape and clothing design based on big data is constructed. After processing with big data methods, human body characteristic data used signals as the input layer of a neural network model and the matching degree management model of human body shape and fashion design. The simulation results show that the built-up model has a matching error of less than $5 \%$, which can effectively improve the matching of human body shape and clothing design.

\section{Introduction}

In fashion design, all functions would be fully displayed on the human body. Based on this, the human body's posture and clothing style composition are complementary and integrated, and they cannot be designed separately [1-3]. Body posture is the theoretical basis of garment style composition, and garment style composition is the ultimate result of showing the smart charm of body posture. Based on the era of big data, the massive growth of data capacity and diversity prompted the use of data mining technology in all areas of mass data analysis. With the increasing demand of personalized clothing design, quickly matching fashion design with human body shape is a beneficial attempt of big data technology and neural network algorithm in the clothing industry. This intelligent fashion design solution can quickly meet the fashion needs of customers [4-6].

Neural network technology has gradually received widespread attention in the garment industry [7]. According to the research, Wang et al. [8] analyzed the apparel style composition under different timing of $\mathrm{BP}$ neural network integration and discussed the application of neural network technology in the apparel industry, including material usage calculation, size recommendation, comfort analysis, etc. Liu et al. [9] applied BP neural network technology to men's suit size range setting, researched and analyzed the factors that affect the proposed model, and obtained the optimal size range setting. But the above model only calculates the data of fashion design, not considering the problem of overfitting in the process of model calculation. So, the resulting error of the fashion design model is large.

Therefore, based on the study of human body shape, the human body index data are collected by the big data method, and the neural network integration method is used to construct the matching degree management model of human body shape and clothing design, so as to realize the automatic mapping matching between human body and clothing design and lay a technical foundation for the mass customization and tailoring of clothing. 


\section{Network Model of Fashion Design}

2.1. Big Data Collection of Human Body Index. Based on the aesthetics of the human body, the images of the human body structure, bone, muscle, shape, and other aspects are collected by the big data method, and the morphological characteristic signals of the upper part of human body are extracted and decomposed into

$$
a=\frac{\beta^{2} \log _{3} \lambda}{\eta}+\sqrt{\beta},
$$

where $\beta$ represents the waist of the human body, $\lambda$ represents the shoulder width of the human body, using centimeters as units, and $\eta$ is the number of sampled signals.

Based on the collected human leg images, the morphological characteristic signals of the lower part of the human body are extracted, and the decomposition formula is as follows:

$$
B=\frac{\mu^{2} \log _{3} \gamma}{\eta}-\gamma,
$$

where $\mu$ refers to the length of human legs and $\gamma$ refers to the data on human height. Combining the morphological characteristics of the upper half of the human body and the legs, the integral surface model is constructed as follows:

$$
c=\frac{\mu^{2} \log _{3} a+\beta^{2}}{\eta}-\log _{2} \tau,
$$

where $\tau$ is the image error caused by the noise. Through this surface model, the data signal of each part of the human body is obtained, and the signal is input into BP neural network as the training sample.

2.2. Garment Data Calculation. The data signals of various parts of the human body were input into the neural network, and $x_{i}$ was set as the basic sample. The physical meanings and dimensions of the data obtained are not uniform, and it is impossible to calculate them. Therefore, the samples need to be normalized [10] before the calculation, so that the data with different measurements can be uniformly processed and the final values of the data are between $[0,1]$ and $[-1,1]$. The specific formula is as follows:

$$
x_{i}^{\prime}=\frac{x_{i}-x_{\min }}{x_{\max }-x_{\min }} .
$$

The body morphological characteristic signals after normalization are input into the hidden layer as the training signal, and the number of nodes in the hidden layer is calculated as follows:

$$
j=\frac{\sqrt{n+m}}{\lambda^{2}}+x_{i}^{\prime}-\log _{2} \partial,
$$

where $n$ is the number of nodes in the input layer, $m$ is the number of nodes in the output layer, and $\partial$ is the number of synapses of neurons. The output of the $j$ node of the hidden layer is as follows:

$$
G_{j}=\sum_{i=1} Q_{i j} x_{i}^{\prime}+\frac{\theta_{j}+Q}{\log _{2} \partial} .
$$

The network weight between the input layer and the hidden layer shall be $Q=q_{1}, q_{2}, \ldots, q_{m} . \theta_{j}$ is the number of neurons in the hidden layer. The signal is transmitted from the hidden layer to the output layer. The number of neurons in the output layer is

$$
Y_{j}=\sum_{j=1} G_{j}+\frac{\varepsilon \sqrt{n+m}}{\log _{2} \partial},
$$

where $\varepsilon$ is the training step. Through nonlinear dynamic calculation of the output layer signal, the activation function $f(x)$ can be expressed as follows:

$$
f(x)=\frac{\varepsilon \sqrt{n+m}}{\log _{2} \partial}-Y_{j}(\omega+\delta),
$$

where $\omega$ is the parameter value of the upper half of the clothing design and $\delta$ is the parameter value of the lower half of the clothing design. Through weighted debugging of formula (8), the formula of network mapping force is as follows:

$$
K=\frac{\varepsilon \sqrt{n+m}}{f(x)+W_{1}}-\sum_{r=1} W_{r} .
$$

The network weight between the hidden layer and the node of the output layer shall be $W=w_{1}, w_{2}, \ldots, w_{r}$. In order to train the body parameter signal samples sufficiently, the dimension of the neural network samples is reduced, and the expressions are obtained as follows:

$$
U=\sum_{r=1} W_{r}-\frac{(T+K) \log _{2} \mu}{f(x)+W_{1}},
$$

where $T$ is the error value in the process of dimensionality reduction. By constructing the data, the neural network model is as follows:

$$
U=\sum_{j=1} W_{j}-\frac{U-\log _{3} \ell}{f(x)+W_{1}},
$$

where $\ell$ is the training error. After many times of training, the value of the minimum node number is determined, and the number of nodes is determined to be 10 . The experimental results are stable, so that the size of the network structure is established to be $12 \times 10 \times 14$. In the range of learning rate between 0.001 and 10 , the optimal value of the final learning rate is determined by successive tests. Experiments show that the learning rate is set to 0.15 , and the maximum number of training steps is 5000; when the sum of squares of errors is 0.001 , the model achieves the best effect.

Based on the above calculation, the neural network topology of fashion design is constructed as shown in Figure 1 .

In Figure 1, $P_{r}$ refers to the output data. The output of the hidden layer is $Y=\left(y_{1}, y_{2}, \ldots, y_{m}\right)^{T}$. 


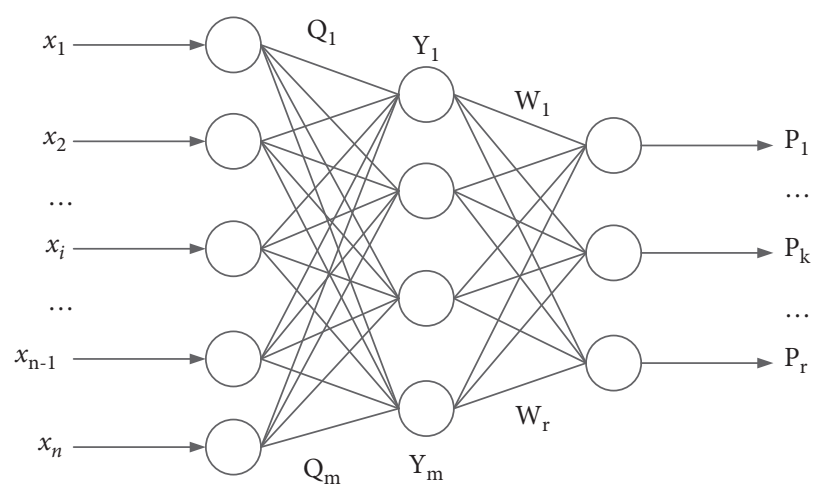

FIGURE 1: BP network topology diagram.

\section{Management Model of Matching Degree between Human Body Shape and Fashion Design}

When there is a simultaneous numerical transformation between the two variables [11-13], that is, when the correlation coefficient between the two variables is larger, then the performance stability of the obtained BP network model will decline, and the reliability of the model will be affected. In order to reduce the error of garment size data, the indexes were screened by the MIV method.

After training with sample $p_{i}$, the original indexes of BP network model increased/decreased by $12 \%$, respectively, and the simulation test is carried out by using the obtained network structure. The result $A_{i}$ is obtained, and the influence degree of each variable on the output result is calculated:

$$
M I V_{i}=\frac{A_{i}-p_{i}}{i}
$$

The neural network calls the mean function in the running process of MATLAB software [14-17]. Select the appropriate variable values for constructing the neural network model. The filtering process is shown in Figure 2.

Screen waist and hip circumference and other variables, rank the results, and compose the MIV datasheet with the top 12 variables, as shown in Table 1.

From Table 1, it can be seen that the trouser length has the greatest influence on the garment pattern structure, while the hip circumference and waist circumference have the second and third place, respectively. Ankle circumference was the least influential variable, and waist length was the second. From the data in Table 1, it can be seen that ankle circumference on the garment pattern structure is negligible. Therefore, the factors of waist length and ankle girth should be removed in order to simplify the optimization procedure of the model.

The parameters are based on the selected body index data and the clothing design data from the neural network. Use the method of correlation analysis to construct the matching model:

$$
V=\left(a_{i} b_{i}-\gamma\right) U+\sum_{j=1} W_{j} c
$$

where $a_{i}$ refers to the morphological parameters of the upper part of the $i$ th human body and $b_{i}$ refers to the lower part of the ith human body. At this point, the matching degree model is constructed.

\section{Model Simulation}

The weak predictor is designed based on BP neural network model, using 5 control points as input layer and using AdaBoost algorithm to construct 10 strong predictors integrated by simple BP neural network.

The experiment selected 500 groups of human data, with 450 groups of human data randomly selected as training samples and the remaining human data selected as a test sample. The upper part of the input layer of BP neural network includes 15 parts related to it, including height, abdominal circumference, front length, back length, etc. The lower part of the input layer of BP neural network includes 10 parts related to it, including height, waist circumference, hip circumference, waist circumference, abdomen circumference, front crotch length, rear crotch length, thigh circumference, and knee height. The output layer of garment type adopts 160/84 form and chooses two values of height, chest circumference, or waist circumference according to the size style. The influence of different parts on height, chest circumference, or waist circumference was obtained accurately. Setting the output node to 1, the upper training curve is shown in Figures 3 and 4, and the lower training curve is shown in Figures 5 and 6.

It can be seen from Figure 3 that in 20 generations of training, the minimum mean square error of height prediction reaches $1 \times 10^{-4}$. As shown in Figure 4, in the 20th generation training, the minimum range of the predicted mean square error of waist circumference is $1 \times 10^{-2} \sim 1 \times 10^{-3}$. From Figure 5, the minimum value of the mean square error of height prediction after 4 generations of training is in the range of $1 \times 10^{-1} \sim 1 \times 10^{-4}$; from Figure 6, the minimum value of the mean square error of waist prediction after 6 generations of training is $1 \times 10^{-1}$.

Through the statistical analysis of the absolute value distribution of the errors of 50 test sets, it can be seen from Figure 7 that the error value of height prediction of the upper model is smaller than that of the ordinary test method, and the larger error fluctuation produced by the prediction 


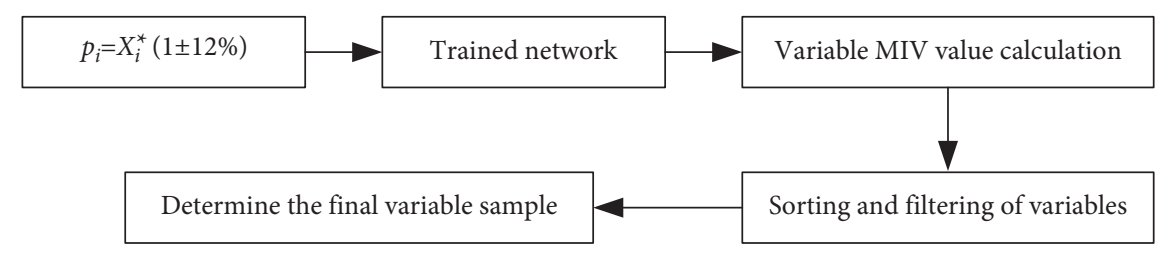

FIGURE 2: Diagram of MIV variable filtering process.

TABLE 1: MIV variable filter sort.

\begin{tabular}{lcc}
\hline Number & Variable name & MIV values \\
\hline 1 & Pant length & 2.6011 \\
2 & Hips & 1.9259 \\
3 & Waist circumference & 0.9357 \\
4 & Inseam length & 0.8534 \\
5 & Calf circumference & 0.8216 \\
6 & Knee circumference & 0.8122 \\
7 & Rise & 0.6137 \\
8 & Waist height & 0.2689 \\
9 & Thigh circumference & 0.2018 \\
10 & Length of upper crotch & 0.1856 \\
11 & Waist length & 0.1754 \\
12 & Ankle circumference & 0.0038 \\
\hline
\end{tabular}

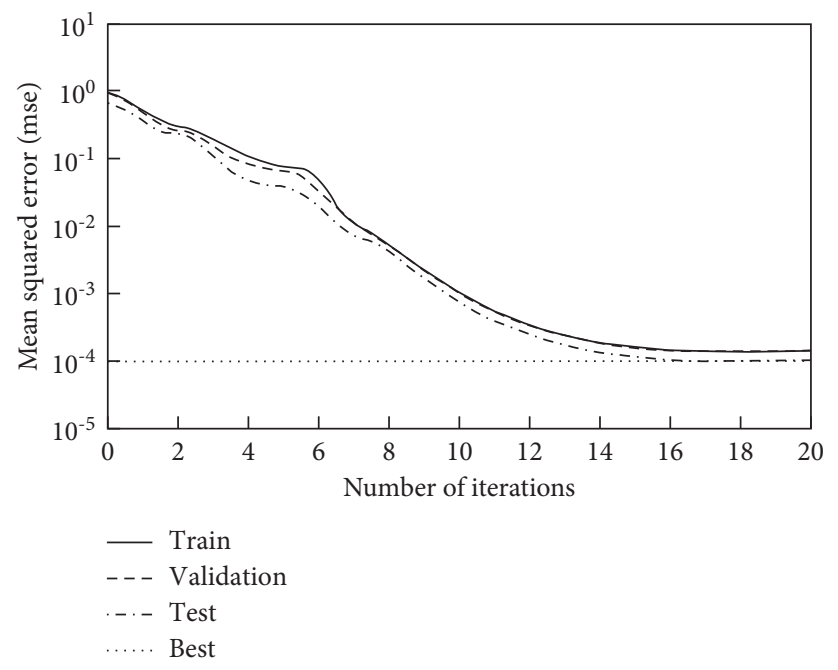

FIgURE 3: Training curve of height prediction model.

sample is in the acceptable range, and the overall absolute error fluctuation range of the quantitative model is less than $3 \%$. As can be seen from Figure 8, the overall absolute error of the experimental model fluctuates slightly, less than $2 \%$, much less than that of the ordinary model, and the range is small.

It can be seen from Figure 9 that the 50 test sets of absolute error distribution statistics in the proposed model are used for error statistics. The maximum absolute error of the ordinary model is close to $4 \%$, while the maximum absolute error of the experimental model is only $1.6 \%$. It proves that the experimental model is relatively stable; from Figure 10, it can be seen that the maximum absolute error of the ordinary model is slightly higher than $7 \%$, while the maximum absolute error of the experimental model does not exceed $2 \%$.

After the comparison of the above data, we can see that the absolute error rate of this experimental method is excellent in the coincidence degrees of predicting height with upper and lower, upper and bust, and lower and waist. 


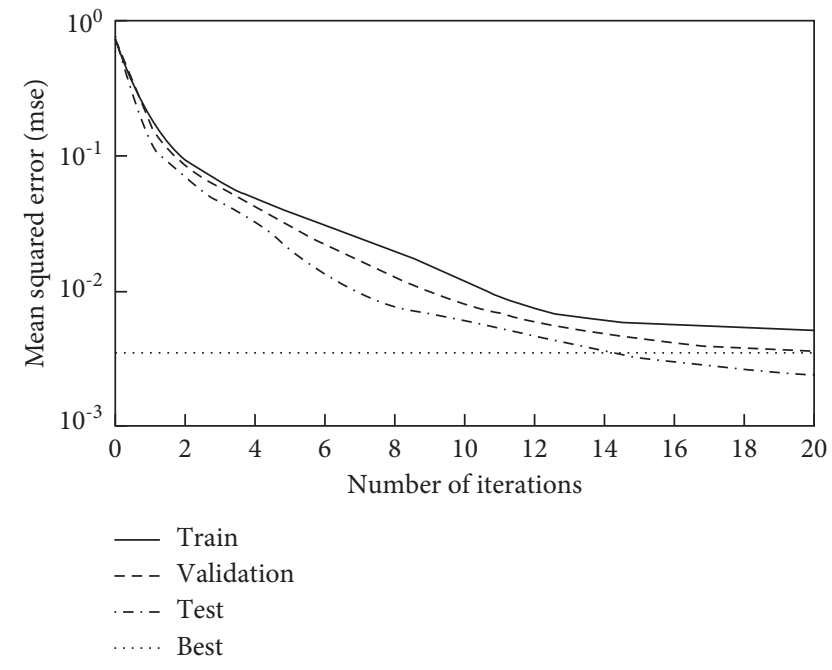

Figure 4: Training curve of predictive model of chest circumference.

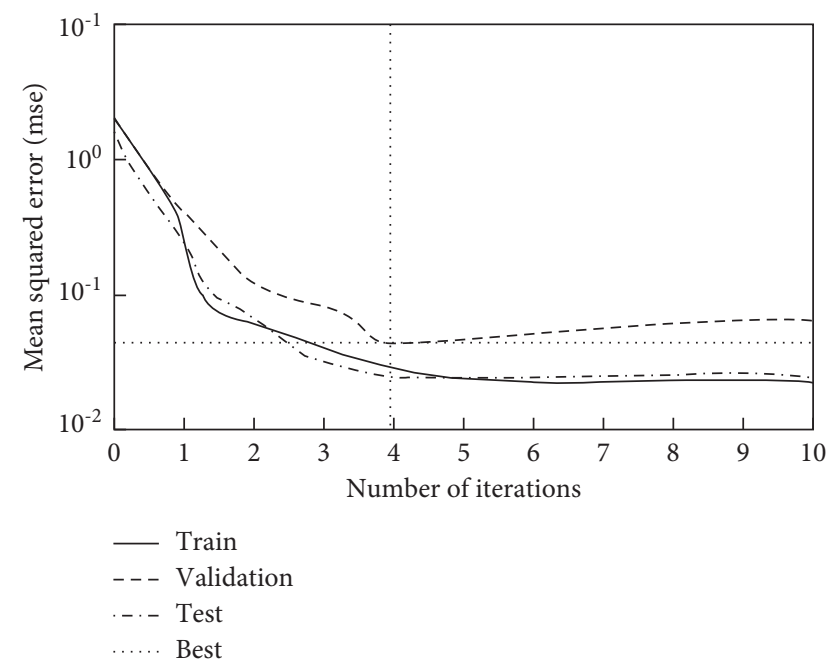

FIgURE 5: Training curve of height prediction model.

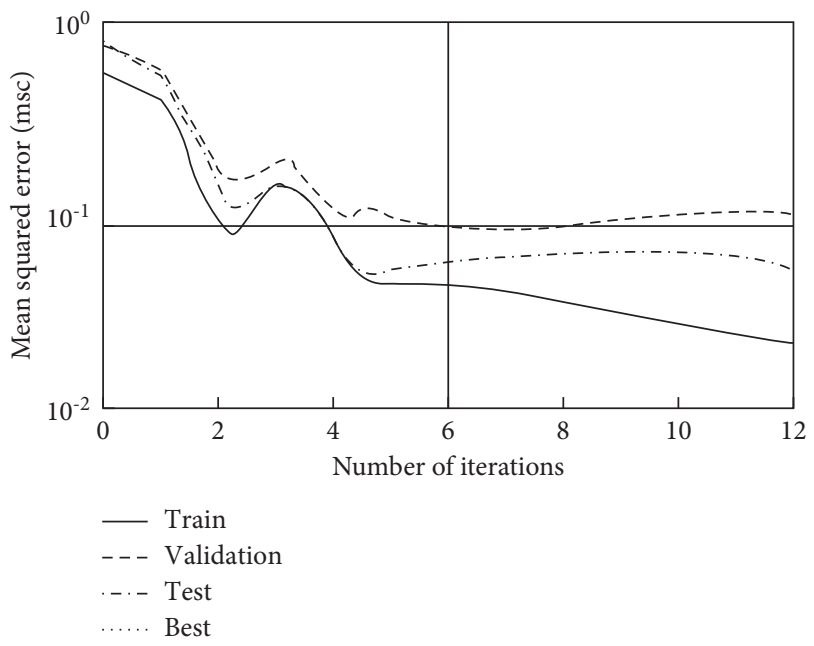

FIgURE 6: Training curve of waistline prediction model.

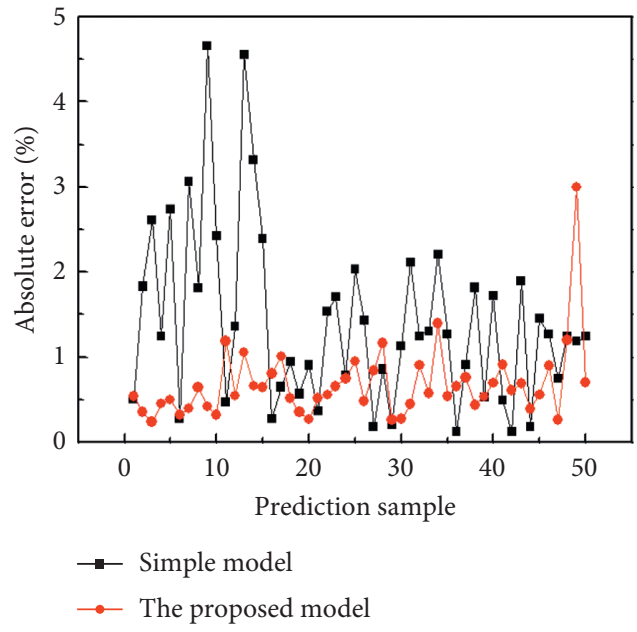

Figure 7: Absolute error of height prediction.

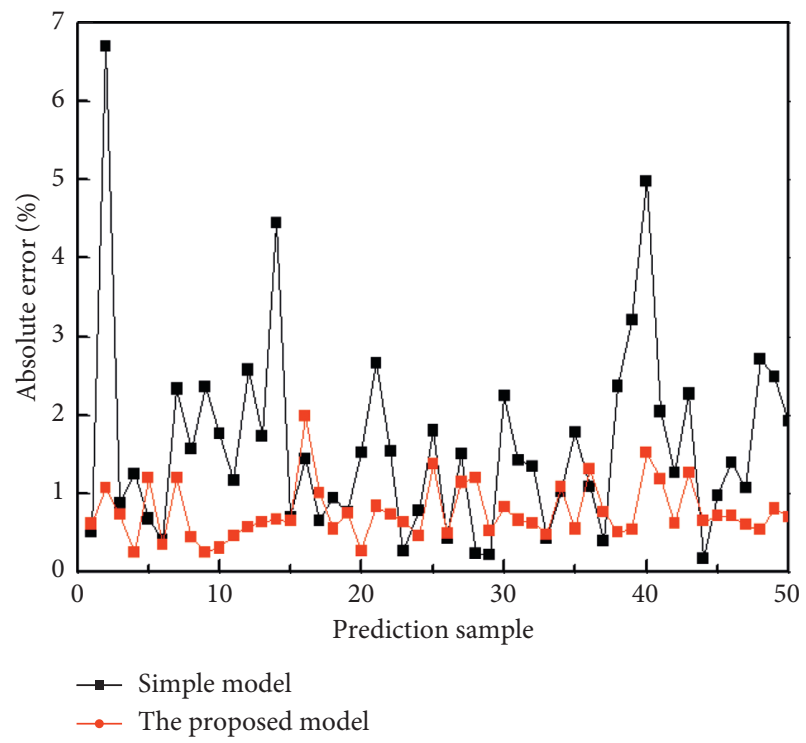

Figure 8: Absolute prediction error of bust size.

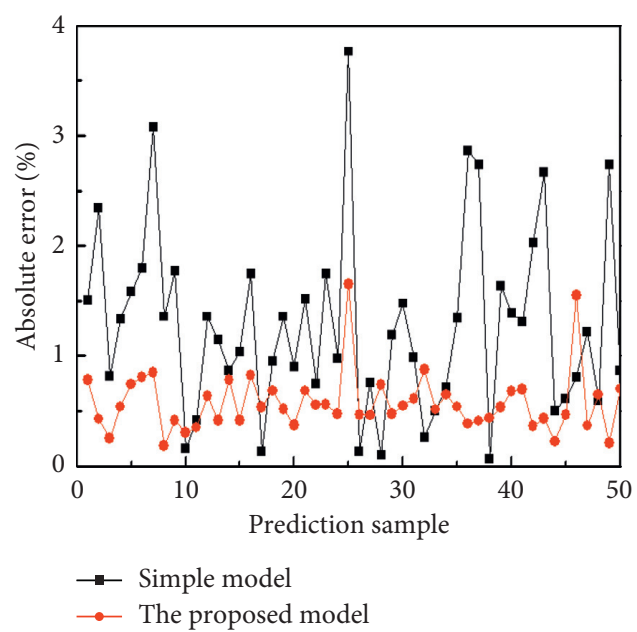

FIGURE 9: Error in height prediction. 


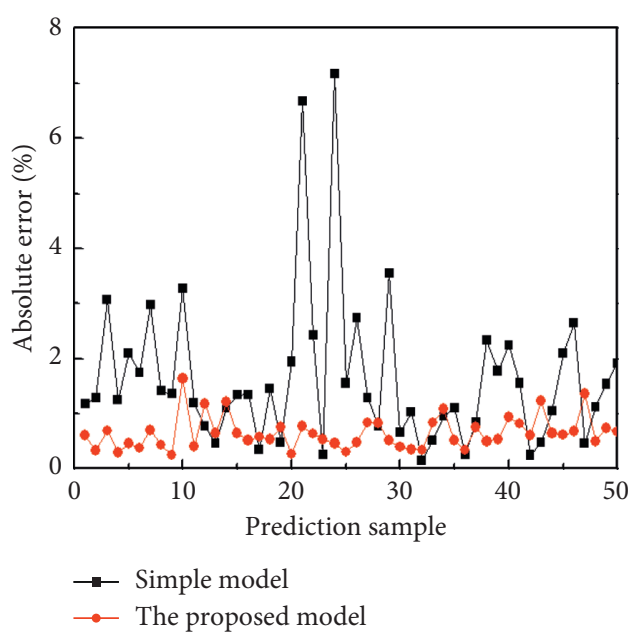

FIgUre 10: Prediction error of belts.

\section{Conclusion}

Based on the analysis of human body shape, this paper constructs the management model of matching degree between human body shape and fashion design. The mapping relationship between human body shape and fashion design is determined by the trial-run experiment, and the prediction model constructed by neural network is simplified by the method of selecting variables. The AdaBoost method is used to integrate $10 \mathrm{BP}$ neural network classification models, and the matching degree management model of human body shape and clothing design based on neural network ensemble is constructed. The matching degree recognition precision of training set is over $95 \%$. The modeling experience of this study shows that the shoulder width and waistline of the human body are vital in clothing design and should get more attention in follow-up studies.

\section{Data Availability}

The data used to support the findings of this study are included within the article.

\section{Conflicts of Interest}

The authors declare that there are no conflicts of interest regarding the publication of this paper.

\section{Acknowledgments}

This study was supported by Educational Science Research Project of Shanghai (no. ZX202007060080) and Talent Studio about Culture and Education of Shanghai.

\section{References}

[1] S. B. Heymsfield, B. Bourgeois, B. K. Ng, M. J. Sommer, X. Li, and J. A. Shepherd, "Digital anthropometry: a critical review," European Journal of Clinical Nutrition, vol. 72, no. 5, pp. 680-687, 2018.

[2] S. C. Hidayati, C. W. You, W. H. Cheng, and K. L. Hua, "Learning and recognition of clothing genres from full-body images," IEEE transactions on cybernetics, vol. 48, no. 5, pp. 1647-1659, 2017.

[3] Y. Asano, K. Okada, and M. Inaba, "Design principles of a human mimetic humanoid: humanoid platform to study human intelligence and internal body system," Science Robotics, vol. 2, no. 13, 2017.

[4] A. Vuruskan and S. P. Ashdown, "Modeling of half-scale human bodies in active body positions for apparel design and testing," International Journal of Clothing Science \& Technology, vol. 29, 2017.

[5] O. Sabina, S. Elena, F. Emilia, and S. Adrian, "Virtual fitting innovative technology for customize clothing design," Procedia Engineering, vol. 69, pp. 555-564, 2014.

[6] S. C. Hidayati, C. C. Hsu, Y. T. Chang, K. L. Hua, J. Fu, and W. H. Cheng, "What dress fits me best? Fashion recommendation on the clothing style for personal body shape," in Proceedings of the 26th ACM international conference on Multimedia, pp. 438-446, 2018 October.

[7] C. Patel, Z. Liao, and G. Pons-Moll, "Tailornet: predicting clothing in $3 \mathrm{D}$ as a function of human pose, shape and garment style," in Proceedings of the IEEE/CVF Conference on Computer Vision and Pattern Recognition, pp. 7365-7375, Seattle, WA, USA, March 2020.

[8] T. Y. Wang, D. Ceylan, J. Popovic, and N. J. Mitra, "Learning a shared shape space for multimodal garment design," 2018, https://arxiv.org/abs/1806.11335.

[9] L. Liu, H. Zhang, Y. Ji, and Q. M. Jonathan Wu, "Toward AI fashion design: an attribute-GAN model for clothing match," Neurocomputing, vol. 341, pp. 156-167, 2019.

[10] A. Perry, L. Malinin, E. Sanders, Y. Li, and K. Leigh, “" perspectives," International Journal of Fashion Design, Technology and Education, vol. 10, no. 3, pp. 372-380, 2017.

[11] A. Kumari, S. Tanwar, S. Tyagi, N. Kumar, M. Maasberg, and K.-K. R. Choo, "Multimedia big data computing and internet of things applications: a taxonomy and process model," Journal of Network and Computer Applications, vol. 124, pp. 169-195, 2018.

[12] B. Roßmann, A. Canzaniello, H. von der Gracht, and E. Hartmann, "The future and social impact of big data analytics in supply chain management: results from a delphi study," Technological Forecasting and Social Change, vol. 130, pp. 135-149, 2018.

[13] A. Urbinati, M. Bogers, V. Chiesa, and F. Frattini, "Creating and capturing value from Big Data: a multiple-case study analysis of provider companies," Technovation, vol. 84-85, pp. 21-36, 2019.

[14] V. Grover, R. H. L. Chiang, T.-P. Liang, and D. Zhang, "Creating strategic business value from big data analytics: a research framework," Journal of Management Information Systems, vol. 35, no. 2, pp. 388-423, 2018.

[15] R. Dubey, A. Gunasekaran, S. J. Childe, S. Fosso Wamba, D. Roubaud, and C. Foropon, "Empirical investigation of data analytics capability and organizational flexibility as complements to supply chain resilience," International Journal of Production Research, vol. 59, no. 1, pp. 110-128, 2021.

[16] X.. Jin, "Deep mining simulation of unstructured big data based on ant colony algorithm," Computer Simulation, vol. 37, no. 11, pp. 329-333, 2020.

[17] Y. Tang and J. Su, "Eye movement prediction based on adaptive BP neural network," Scientific Programming, vol. 2021, Article ID 4977620, 9 pages, 2021. 